\title{
El Liderazgo Distribuido para la Mejora Educativa: Análisis de Redes Sociales en Departamentos de Escuelas Secundarias Chilenas
}

\author{
Distributed Leadership for Educational Improvement: A Social \\ Network Analysis in Chilean High Schools' Departments
}

\author{
Juan Pablo Queupil 1* \\ Carmen Montecinos ${ }^{2}$ \\ ${ }^{1}$ Universidad Católica Silva Henríquez, Chile \\ ${ }^{2}$ Pontificia Universidad Católica de Valparaíso, Chile
}

\begin{abstract}
La mejora educativa involucra interacciones entre individuos en contextos escolares, lo cual conlleva roles de liderazgo y su eminente distribución. En este estudio exploratorio-descriptivo usamos el Análisis de Redes Sociales (ARS) en una muestra de tres escuelas secundarias de Chile para examinar las relaciones entre los miembros de los departamentos de Lenguaje y Matemáticas, identificando quienes ejercen roles de liderazgo para los procesos de mejora educativa. Los resultados indican que los patrones son variados según el tipo de dimensión de colaboración, observando escuelas en las cuales las interacciones son escasas entre y dentro de los departamentos analizados, y en otras más intensas y frecuentes. Si bien el liderazgo de los jefes de departamento es importante, en algunos casos los docentes que no tienen ese cargo pueden incluso tener un papel más preponderante en influenciar procesos de mejora escolar. Todo lo anterior permite concluir que la técnica del (ARS) posibilita representar el capital social de una escuela para la mejora educativa, identificando aspectos a fortalecer para incidir en una mayor distribución del liderazgo y su impacto en la mejora escolar.
\end{abstract}

Descriptores: Liderazgo; Mejora escolar; Redes sociales; Escuelas secundarias; Chile.

Educational improvement involves interactions among individuals in school contexts, which entails leadership roles and their potential distribution. In this exploratory-descriptive study we use Social Network Analysis (SNA) with a sample of three secondary schools in Chile to examine the relationships between members of the Language and Mathematics departments, identifying those who play leadership roles for educational improvement processes. Findings show that patterns of interactions among teachers varied by type of collaborative action, where in some schools the interactions among teachers are scarce between and within the departments analyzed, and in others they are more intense and frequent. Whereas the role of department heads is important, in some cases teachers who do not have that position may even have a more prominent role in influencing school improvement processes. Findings lead us to conclude that SNA is a useful technique for depicting a school's social capital, enabling the identification of aspects in need of attention to increase distributed leadership as leverage for school improvement.

Keywords: Leadership; School improvement; Social network analysis; Secondary schools; Chile.

*Contacto: jqueupil@ucsh.cl

ISSN: 1696-4713

www.rinace.net/reice/

revistas.uam.es/reice
Recibido: $\quad 2$ de octubre 2019

$1^{\text {a }}$ Evaluación: 14 de diciembre 2019

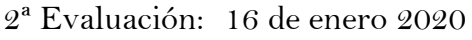

Aceptado: 1 de febrero 2020 


\section{Introducción}

Alrededor del mundo, la mejora educativa es una de las problemáticas que ha recibido mayor atención en las últimas décadas, principalmente impulsada por una serie de reformas que han tenido resultados disímiles (Martínez-Valdivia, García-Martínez e Higueras-Rodríguez, 2018; Murillo y Krichesky, 2015). En ese sentido, Hallinger y Heck (2014), entre otros autores, resaltan que el liderazgo ciertamente contribuye a la capacidad de los establecimientos educativos para la mejora escolar. Asimismo, un estudio de Leithwood y sus colaboradores (2006) concluyó que el liderazgo total en las escuelas representaba el $27 \%$ de la variación entre escuelas respecto de los resultados de aprendizaje.

Para incrementar la capacidad de mejora escolar se apuesta por un liderazgo distribuido que posibilite el logro de metas organizacionales a través de acciones colectivas (Harris, 2004; Hopkins, Ainscow y West, 1994). Este liderazgo promueve la mejora de la enseñanza en el aula, ya que se basa en el apoyo, estimulación e influencia en él y del profesorado (Hallinger y Heck, 2014). En particular, se ha detectado la importancia de estructuras que posibilitan la colaboración entre docentes y de las redes sociales para promover el desarrollo profesional de los docentes (Daly et al., 2010; Murillo y Krichesky, 2015).

En escuelas secundarias, las experiencias de colaboración profesional para la gran mayoría de los docentes están circunscritas sustancialmente por los departamentos disciplinarios, ya que en estas estructuras organizativas se despliegan sus interacciones y desarrollo profesional (Harris, 2000, 2001; Siskin y Little 1995). En la literatura, los jefes de departamento se reconocen como líderes medios, ya que, si bien no son integrantes del equipo directivo, tienen responsabilidades formales en la gestión pedagógica de un grupo de profesores. Esta distribución del liderazgo pedagógico reconoce que quien mejor conoce las prácticas pedagógicas relevantes a un área disciplinaria son los especialistas y, por lo tanto, la intervención de los jefes de departamento puede tener más impacto en comparación al liderazgo del director o directora (Robinson, 2007). Así, los jefes de departamento pueden desempeñar un papel clave en la mejora educativa, sobre todo en el desarrollo y promoción de departamentos efectivos en términos del logro estudiantil (Wenner y Campbell, 2017).

El presente estudio examina el liderazgo en la estructura departamental a través de un análisis cuantitativo de las interacciones que promueven la colaboración en procesos de mejora escolar, el intercambio de prácticas para mejorar la enseñanza y la innovación pedagógica. Utilizando un enfoque empírico de análisis de redes sociales (ARS), se calculan métricas y grafican patrones de interacciones relacionadas con prácticas que promueven la mejora en los departamentos de Lenguaje y Matemáticas de una muestra de tres escuelas secundarias chilenas. Este análisis permite identificar patrones a través de los cuales las ideas, información, recursos e influencia fluyen en los departamentos y entre departamentos (Daly, 2010). 


\section{Revisión de la literatura}

\subsection{El liderazgo distribuido y su papel en la mejora educativa}

La evidencia sobre mejora escolar destaca la responsabilidad colectiva y la actividad colegiada de grupos amplios de docentes, incorporando la noción de liderazgo distribuido (Frost y Harris, 2003). Algunas definiciones subrayan este liderazgo como aquel que minimiza intencionalmente, o elimina, diferencias de poder e influencia entre quienes ocupan cargos de autoridad formal y los otros actores del escenario educativo (Barry, 1991). De acuerdo con Spillane (2006), una visión distribuida del liderazgo implica centrarse en las redes de relaciones de los actores, así como en sus situaciones y prácticas. Desde esta perspectiva, la exploración del liderazgo debe evitar hacer suposiciones a priori con respecto a la distribución de las funciones entre quienes ocupan cargos formales. Más bien, el objeto de estudio son las interacciones entre los individuos, enfocado en el desarrollo de capacidad de influencia mutua (Garay et al., 2019; Gronn 2002; Harris, 2001).

Existe un creciente consenso que el liderazgo distribuido produce un impacto positivo en los resultados escolares y promueve una mejora educativa exitosa (Camburn, Rowan, y Taylor, 2003; Day y Harris 2002; Gronn, 2002; Harris, 2013; Spillane, 2006; Wallace, 2002). Distribuir el liderazgo en los establecimientos educativos implica valorar la experticia que aportan distintos docentes para el logro de las metas institucionales. Así, la distribución del liderazgo pedagógico se define como la capacidad de docentes líderes para involucrar a sus colegas en el desarrollo profesional mutuo y colaborativo, con el fin de mejorar los procesos de enseñanza y aprendizaje (Aubrey-Hopkins y James, 2002; Bennett, 1995; Busher, Harris, y Wise , 2001).

\subsection{Liderazgo en los departamentos disciplinares y rol del jefe de departamento}

Una forma de abordar el estudio del liderazgo distribuido en las escuelas es centrarse en los puestos de liderazgo formalmente designados. En este estudio, nos focalizamos en los jefes de departamentos, en su calidad de líder pedagógico medio, que articula entre las orientaciones de mejoramiento a nivel escolar y las respuestas y necesidades docentes. Siguiendo a Wenner y Campbell (2017), el docente líder es quien cumple funciones de enseñanza y aprendizaje con estudiantes y, además, asume responsabilidades fuera del aula que han sido asignadas desde la estructura de la organización escolar.

Los líderes pedagógicos medios proveen una instancia de alineamiento y control que antes era privativa del equipo directivo (Bush, 2003). Flückiger y sus colegas (2015) identifican las tareas de los jefes de departamento: (a) comprometer a los docentes hacia prácticas colaborativas, (b) potenciar conversaciones reflexivas enfocadas en formas de innovar y mejorar, (c) observar y retroalimentar prácticas, (d) modelar buenas prácticas, y (e) comprender los sistemas de rendición de cuentas. Para llevar a cabo estas tareas, configuran un trabajo colaborativo entre docentes del departamento que posibilite acceder y utilizar los recursos individuales y colectivos que están disponibles en la comunidad escolar (Daly et al., 2010).

Hasta la fecha se han realizado escasas investigaciones sobre este tema específico para establecimientos educativos chilenos, por lo cual no es claro si los jefes de departamento ejercen liderazgo efectivo para la mejora educativa y en qué medida está presente una distribución de liderazgo en los departamentos (Cortez y Zoro, 2016). En un estudio de 
caso de un establecimiento secundario chileno, Araya (2015) observó que las funciones del jefe de departamento incluían acciones tales como: planificar, ejecutar, supervisar y evaluar las actividades docentes y de perfeccionamiento, comunicar a los integrantes de su departamento las informaciones necesarias para su buena marcha, y proponer y apoyar actividades de enriquecimiento curricular beneficio de los estudiantes.

Araya (2015) no examinó los patrones de interacción entre los jefes de departamento y sus colegas en el despliegue de estas funciones. Así, no hay evidencia empírica respecto a cómo los jefes de departamento establecen vínculos profesionales dentro y entre departamentos, a fin de promover la mejora escolar. Analizar las interacciones de docentes que son reconocidos como influyentes merece atención por su potencial impacto en el desempeño profesional de los docentes y, en consecuencia, en cuánto aprenden los estudiantes (De Lima, 2008).

\subsection{El análisis de redes sociales para examinar el liderazgo distribuido en departamentos de establecimientos educativos}

Mediante la recopilación de información sobre las relaciones de cada miembro de los departamentos con los demás integrantes del equipo docente y directivo, se puede comprender el contexto social inmediato del liderazgo departamental. Metodológicamente, esta es una manera apropiada de identificar la distribución del liderazgo, no solo entre los líderes designados sino también entre los líderes que aparecen en la evidencia práctica, es decir, las personas que, independientemente de su posición, ejercen alguna influencia sobre sus colegas (Spillane, 2006). En otras palabras, la naturaleza multidireccional e interactiva del liderazgo docente distribuido en las escuelas requiere la utilización de un enfoque de investigación de redes sociales que devele el entramado y los patrones de relaciones que existen dentro y entre departamentos.

En este sentido, el análisis de redes sociales (ARS) se basa en el supuesto que las relaciones entre personas representan un recurso -el capital social- de las organizaciones escolares para movilizar el cambio y la mejora (Downey, 2018). En particular, dos conceptos del análisis de redes sociales son particularmente importantes para comprender los patrones dentro y entre departamentos y grupos de docentes. Una es la densidad de red, que se refiere a la cantidad de vínculos presentes que existen en una red dada, en comparación con el total de vínculos potenciales que son teóricamente posibles en esa red (Wasserman y Faust, 1994). Un sistema de liderazgo distribuido implica una alta densidad, pues habrá varios docentes -en roles formales como informales-, y no solo uno, que serán recurridos y considerados por sus colegas, y también porque estos líderes serán activos y efectivos en interactuar y colaborar con otros pares para la mejora educativa de sus departamentos y sus establecimientos.

El otro concepto relevante es la centralidad, una medida que describe la prominencia o el prestigio de un actor dentro de una red (Borgatti y Everett, 2006; Wasserman y Faust, 1994). Así, los docentes líderes son aquellos actores relevantes en sus redes porque están involucrados en muchas interacciones, colaboraciones o lazos profesionales con otros colegas.

El presente estudio tiene como objetivo examinar qué tan centralizados o distribuidos están los roles y liderazgos de los miembros de los departamentos de Lenguaje y Matemáticas -en particular, sus respectivas jefaturas- en los procesos y prácticas colaborativas para la mejora y transformación educativa. En otras palabras, el ARS permite 
establecer en qué medida se ejercen las funciones de liderazgo y cómo se distribuyen tanto a los jefes de departamentos como a otros colegas docentes a través de sus interacciones.

\section{Método}

Los datos que se reportan en este artículo se han producido en el contexto de una investigación más amplia que examina la contribución de los jefes de departamento a la mejora escolar, utilizando un diseño de estudio de casos múltiples (Merriam, 1988). Utilizando técnicas del análisis de redes sociales (ARS) este estudio tiene un enfoque cuantitativo para visualizar las interacciones profesionales de los docentes de los departamentos de Lenguaje y Matemáticas (Scott, 2000; Wasserman y Faust, 1994). A través de la construcción de sociogramas, ARS permite identificar los patrones de liderazgo que emergen de estas interacciones (Friedkin y Slater, 1994; Hadfield y Jopling, 2012; Hoppe y Reinelt, 2010).

\section{Muestra}

Dado el carácter exploratorio-descriptivo de la investigación, la muestra del estudio son tres escuelas secundarias en Chile (catalogados como Escuelas Agua, Cielo, y Tierra ${ }^{1}$ ), ubicadas en una de las tres regiones más pobladas del país (V, VIII y Región Metropolitana). En cada escuela se invitó a participar en el estudio a los docentes de los departamentos de Lenguaje y Matemáticas, incluyendo sus jefaturas. Estas escuelas tienen una cantidad similar de docentes totales de Lenguaje y Matemática $(10,11$ y 12, respectivamente).

El principal criterio de selección de estos establecimientos fue su trayectoria de mejora en la enseñanza secundaria en la última década, utilizando el índice de desempeño educativo (IDE) (Valenzuela, Bellei y Allende, 2016). Este considera un amplio conjunto de indicadores de resultados de los establecimientos del país, y ha sido utilizado con éxito en otros estudios focalizados en la educación primaria chilena (Bellei, Valenzuela, Vanni y Contreras, 2014). La selección de escuelas efectivas da cuenta del objetivo general del estudio más amplio y recoge la evidencia empírica que muestra, entre otros aspectos, que estas se caracterizan por un liderazgo pedagógico fuerte, docentes con altos niveles de auto eficacia y comprometidos con la mejora escolar (Wang, Walters y Thum, 2013).

\section{Técnicas e instrumentos de producción de datos}

Se elaboró un cuestionario con tres "preguntas de red" en base a otras utilizadas en diversas investigaciones de redes sociales en contextos escolares (Moolenaar, 2012; Smith, Trygstad y Hayes, 2018), las cuales fueron previamente piloteadas para el contexto chileno. En particular, se preguntó la frecuencia con la cual recurren a otros colegas con relación a tres dimensiones de colaboración: a) trabajar en procesos de mejora escolar en su disciplina, b) pedir consejo sobre cómo mejorar su enseñanza, y c) obtener prácticas innovadoras. Para cada dimensión, se entregó una matriz con los nombres de todos los docentes de la escuela en las filas y la frecuencia de interacciones en las columnas (desde $0=$ Nunca hasta $4=$ Casi a diario). Luego de firmar un consentimiento informado 
accediendo a participar voluntariamente en el estudio, todos los docentes de estas escuelas completaron las tres matrices durante el año 2018.

\section{Análisis de datos}

Diversos conceptos y medidas de red destacados en la literatura previa para el liderazgo docente distribuido se pusieron en práctica en el estudio. Una red está compuesta de actores -que en el lenguaje de ARS se denominan nodos- e interacciones, las que técnicamente se llaman enlaces o links (Wasserman y Faust, 1994). Estas dos nociones se pueden visualizar en mapas de red o sociogramas, donde los nodos son círculos y las interacciones son flechas, lo cual conlleva una serie de métricas que son aplicadas a este estudio (Scott, 2000).

De acuerdo con lo señalado por Moolenaar (2012), la principal métrica utilizada para examinar las características del liderazgo de una red a nivel de actores -en este caso, docentes- es el grado nodal (degree centrality), el cual es generalmente representado visualmente con flechas que van hacia el actor, convirtiéndose en in-degree centrality (número de nominaciones que recibe un actor) o flechas que salen del actor, lo que se denomina out-degree centrality, cuando las relaciones no son simétricas, como es el caso de este estudio (si el docente A nomina al docente B, no implica que el docente B nomina al docente A). Así, el grado nodal es una medida de la "popularidad" de un actor; en otras palabras, indica las conexiones directas de un actor a otros miembros de la red (Wasserman y Faust, 1994).

En este caso, para cada tipo de relación, se determinó el grado de centralidad (in-degree y out-degree) para cada docente. Así, un alto puntaje de centralidad indica que el docente en cuestión estuvo involucrado con muchos colegas en relaciones profesionales dentro y/o entre departamentos. Para cada tipo de relación bajo análisis, todos los actores en cada departamento -Lenguaje y Matemáticas- fueron anonimizados y ordenados según centralidad in-degree para la dimensión procesos de mejora.

La frecuencia de las interacciones que fue recolectada en los cuestionarios fue utilizada para construir el sociograma de cada departamento. Como se mencionó previamente, un sociograma es un mapa donde se puede visualizar los actores de la red y las relaciones presentes entre los actores (Smith et al., 2018). En este caso, una flecha más gruesa representa una relación más frecuente entre dos actores específicos.

A nivel de la red como un todo, se utilizó la métrica de densidad de red, la cual indica la proporción de relaciones que están realmente presentes en una red, en relación con el total de relaciones que son teóricamente posibles. Las densidades de red varían de cero $(0 \%)$ (no existe ninguna de las relaciones teóricamente posibles) a uno (100\%) (se ejecutan todas las relaciones posibles). Así, la densidad puede verse como la plenitud de la red, es decir, el grado en que todas las relaciones potenciales están realmente presentes (Wasserman y Faust, 1994). Para este estudio, bajas densidades indican una falta de distribución del liderazgo dentro y/o entre departamentos (De Lima, 2008).

En cuanto a los sociogramas, para este estudio, el tamaño de los nodos refleja el grado indegree de cada docente, dimensionando el número de nominaciones o flechas que van hacia ellos. A su vez, los sociogramas pueden mostrar la red general, pero también varios subgrupos o clústeres, es decir, regiones locales en una red con densidad relativamente alta y relativamente pocos enlaces a otro subconjunto de nodos (Wasserman y Faust, 1994). Para este estudio, la identificación de clústeres se hizo a partir del cálculo Wakita-Tsurumi 
(Wakita y Tsurumi, 2007). Dicho cálculo identifica "vecindarios" de colaboración que se definen por los patrones y frecuencias de las interacciones que tienen como resultado una alta densidad dentro de un subconjunto de nodos. Se hipotetizó que cada departamento curricular aparecería como un clúster específico, lo cual, de ser así, el análisis lo especificará con colores diferentes. Todos los datos se analizaron con las métricas de centralidad, la densidad y diagramas de sociogramas y clústeres incluidas en el programa NodeXL (Smith et al., 2010).

\section{Resultados}

\subsection{Densidad}

Los resultados de este estudio indican diferentes grados de densidad de las redes relacionadas con la distribución del liderazgo en los contextos analizados. En primer lugar, las densidades de las relaciones presentes para las tres dimensiones de colaboración varían siguiendo una tendencia decreciente. Las densidades más altas se encuentran en las relaciones referidas a "procesos de mejora en la disciplina", seguido de "pedir consejo sobre cómo perfeccionar clases”, y finalmente por "obtención de prácticas innovadoras” (ver cuadro 1).

Cuadro 1. Densidades de las redes según escuela secundaria, departamento y dimensión de colaboración

\begin{tabular}{lccc}
\hline & \multicolumn{3}{c}{ EsCUELA } \\
\cline { 2 - 4 } & Agua & Cielo & Tierra \\
\hline Región & $\mathrm{V}$ & VIII & RM \\
Total docentes encuestados & 10 & 11 & 12 \\
Total docentes Lenguaje & 6 & 5 & 6 \\
Total docentes Matemáticas & 4 & 6 & 6 \\
Densidad "P. Mejora" (\%) & 49 & 41 & 57 \\
Densidad "Consejo" (\%) & 31 & 30 & 31 \\
Densidad "P. Innovadoras" (\%) & 36 & 24 & 31 \\
\hline Fuente: Elaboración propia.
\end{tabular}

Fuente: Elaboración propia.

Las densidades en lo referente a interacciones entre docentes para los procesos de mejora siempre supera el $40 \%$ e incluso puede bordear el $60 \%$. Aquellas interacciones que involucran algún tipo de transformación educativa a nivel más individual, tales como a quién recurrir para perfeccionar clases u obtener prácticas innovadoras, bordean el 30\% en términos de densidad. Otro aspecto interesante de notar es que no es del todo claro si existe una relación inferencial entre el total de los docentes de las escuelas y las densidades, por lo que no es evidente que escuelas con menos docentes en Lenguaje y Matemática sean más cohesionados o viceversa.

\subsection{Centralidad}

Los cuadros 2, 3 y 4 presentan los grados de centralidad de los docentes de Lenguaje y Matemáticas -incluidas sus jefaturas- para las escuelas Agua, Cielo y Tierra, respectivamente. Estos están ordenados de acuerdo con el grado de centralidad in-degree de la dimensión "procesos de mejora en la disciplina". Las figuras 1, 2, y 3 presentan los sociogramas asociados. Dado el carácter exploratorio del estudio, se presenta ilustrativamente un solo sociograma para cada dimensión y caso analizado: la figura 1 
presenta el sociograma del Escuela Agua para la red de la dimensión procesos de mejora; la figura 2 muestra el sociograma de la Escuela Cielo para la red de la dimensión consejo para mejorar la enseñanza; y la figura 3 exhibe el sociograma de la Escuela Tierra para la red de la dimensión prácticas innovadoras. Cabe recordar que los docentes (nodos) han sido anonimizados, y cada color de nodo representa un clúster o sub-grupo diferente, de acuerdo al análisis aplicado en cada sociograma.

El cuadro 2 indica que la jefatura de Lenguaje (AG) en la Escuela Agua está entre las más recurridas en las dimensiones analizadas, complementado por el destacado rol de otros docentes del área, tales como el docente A, quien recibe 6 nominaciones de un máximo posible de 9 colegas. Incluso más, AG es quien suele hacer más menciones a otros colegas. Algo similar pasa con la jefatura de Matemáticas $(G)$, pero su departamento en sí presenta menos interacciones, en particular por el rol de $\mathrm{J}$, quien no hace menciones a otros colegas. A pesar de ello, la red presenta una alta densidad, sobre todo en lo que respecta a las interacciones relativas a "procesos de mejora en la disciplina", lo cual se visualiza en la figura 1.

Cuadro 2. Indicadores de centralidad en la Escuela Agua

\begin{tabular}{llcccccc}
\hline & & \multicolumn{5}{c}{ DIMENSIONES DE COLABORACIÓN } \\
\cline { 3 - 7 } NoDo & \multirow{2}{*}{ CARGo } & \multicolumn{2}{c}{ P. Mejora } & \multicolumn{2}{c}{ Consejo } & \multicolumn{2}{c}{ P. Innovadoras } \\
\cline { 3 - 7 } & & $\begin{array}{c}\text { In- } \\
\text { Degree }\end{array}$ & $\begin{array}{c}\text { Out- } \\
\text { Degree }\end{array}$ & $\begin{array}{c}\text { In- } \\
\text { Degree }\end{array}$ & $\begin{array}{c}\text { Out- } \\
\text { Degree }\end{array}$ & $\begin{array}{c}\text { In- } \\
\text { Degree }\end{array}$ & $\begin{array}{c}\text { Out- } \\
\text { Degree }\end{array}$ \\
\hline A & Docente Lenguaje & 6 & 5 & 6 & 5 & 4 & 5 \\
L & Docente Lenguaje & 6 & 5 & 2 & 5 & 3 & 5 \\
P & Docente Lenguaje & 6 & 6 & 3 & 2 & 4 & 3 \\
W & Docente Lenguaje & 6 & 5 & 3 & 1 & 4 & 0 \\
AG & Jefatura Lenguaje & 5 & 6 & 3 & 5 & 4 & 5 \\
V & Docente Lenguaje & 5 & 6 & 3 & 1 & 4 & 5 \\
G & Jefatura Matemáticas & 3 & 4 & 2 & 4 & 2 & 3 \\
J & Docente Matemáticas & 3 & 0 & 2 & 0 & 3 & 0 \\
M & Docente Matemáticas & 2 & 2 & 2 & 2 & 2 & 3 \\
U & Docente Matemáticas & 2 & 5 & 2 & 3 & 2 & 3 \\
\hline
\end{tabular}

Fuente: Elaboración propia.

En la figura 1 destaca que el análisis de clústeres sí arroja dos departamentos separados (Lenguaje en azul oscuro y Matemáticas en azul claro). Además, resalta el rol de ambas jefaturas de departamento (AG y G, para Lenguaje y Matemáticas, respectivamente). Se observa que las interacciones son frecuentes (reflejadas en el grosor de las flechas) y a su vez existe una interacción interesante entre los departamentos cuando se abordan procesos de mejora.

En cuanto a la Escuela Cielo, el cuadro 3 indica un liderazgo relevante de la jefatura de Matemáticas para todas las dimensiones analizadas, y no así para la jefatura de Lenguaje, la cual va siendo menos relevante para las dimensiones "consejo" y "prácticas innovadoras". Para la dimensión "pedir consejo sobre cómo perfeccionar clases", hay tres docentes que no reciben nominaciones y dos de ellos están entre los que realizan más menciones (BJ y BK, docentes de Lenguaje y Matemáticas, respectivamente). 


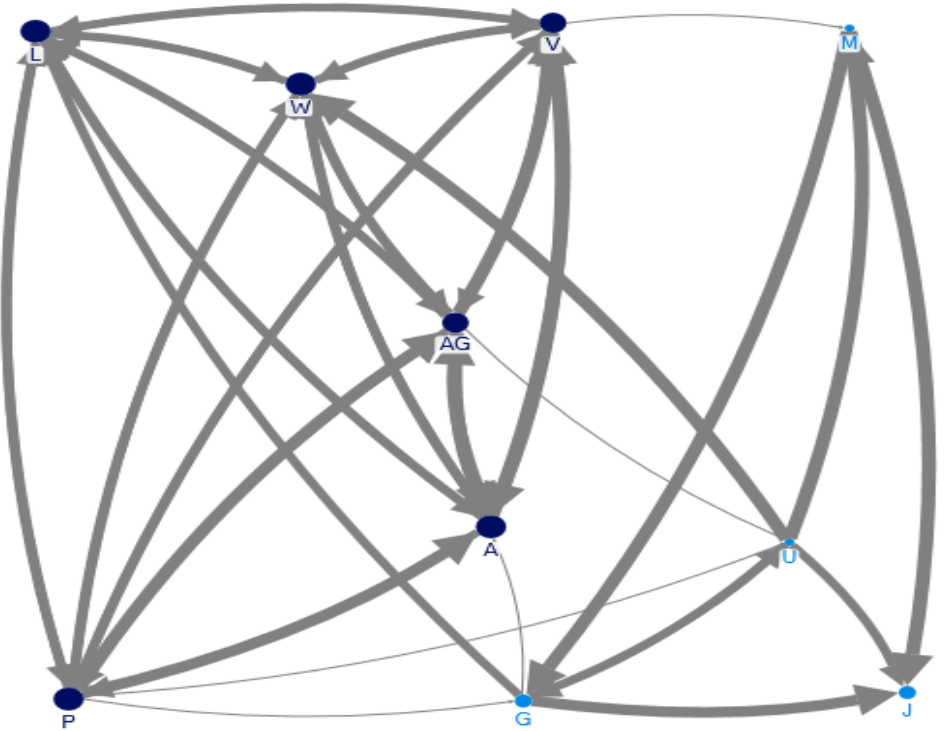

Figura 1. Sociograma de relaciones en "procesos de mejora en el área” para la Escuela Agua

Fuente: Elaboración propia.

Cuadro 3. Indicadores de centralidad en la Escuela Cielo

\begin{tabular}{llcccccc}
\hline & & \multicolumn{5}{c}{ DIMENSIONES DE COLABORACIÓN } \\
\cline { 3 - 7 } NoDo & \multirow{2}{*}{ CARGo } & \multicolumn{2}{c}{ P. Mejora } & \multicolumn{2}{c}{ Consejo } & \multicolumn{2}{c}{ P. Innovadoras } \\
\cline { 3 - 7 } & & $\begin{array}{c}\text { In- } \\
\text { Degree }\end{array}$ & $\begin{array}{c}\text { Out- } \\
\text { Degree }\end{array}$ & $\begin{array}{c}\text { In- } \\
\text { Degree }\end{array}$ & $\begin{array}{c}\text { Out- } \\
\text { Degree }\end{array}$ & $\begin{array}{c}\text { In- } \\
\text { Degree }\end{array}$ & Out- \\
& & 8 & 4 & 6 & 4 & 6 & 3 \\
\hline K & Jefatura Matemáticas & 7 & 5 & 6 & 3 & 3 & 0 \\
BC & Docente Matemáticas & 7 & 3 & 5 & 3 & 4 & 3 \\
L & Docente Matemáticas & 6 & 3 & 4 & 2 & 4 & 0 \\
J & Docente Matemáticas & 5 & 5 & 5 & 3 & 4 & 2 \\
AE & Docente Matemáticas & 5 & 2 & 3 & 2 & 3 & 2 \\
AI & Jefatura Lenguaje & 4 & 4 & 3 & 2 & 3 & 2 \\
AA & Docente Lenguaje & 1 & 6 & 1 & 2 & 0 & 4 \\
AH & Docente Lenguaje & 1 & 2 & 0 & 2 & 0 & 1 \\
BI & Docente Lenguaje & 1 & 6 & 0 & 5 & 0 & 5 \\
BJ & Docente Lenguaje & 0 & 5 & 0 & 5 & 0 & 5 \\
BK & Docente Matemáticas & & & & \\
\hline
\end{tabular}

Fuente: Elaboración propia.

Observando la figura 2, es posible notar nuevamente una separación de clústeres (Lenguaje en azul claro y Matemáticas en azul oscuro). Sin embargo, BJ, que es docente de Lenguaje, pasa a formar parte del clúster de Matemáticas, pues solo recurre a docentes de esa área. Incluso más, este docente no es recurrido por ningún colega, ni de Lenguaje ni de Matemáticas. Por otro lado, las frecuencias de interacción entre los departamentos son bajas y están prácticamente separados, de no ser por el vínculo entre K y AI, recordando que estos son jefaturas de departamento. Además, se observa una mayor cohesión en el departamento de Matemáticas, destacando el rol de prácticamente todos sus docentes, a pesar de que BK es uno de lo que realiza más nominaciones, pero no recibe ninguna. 


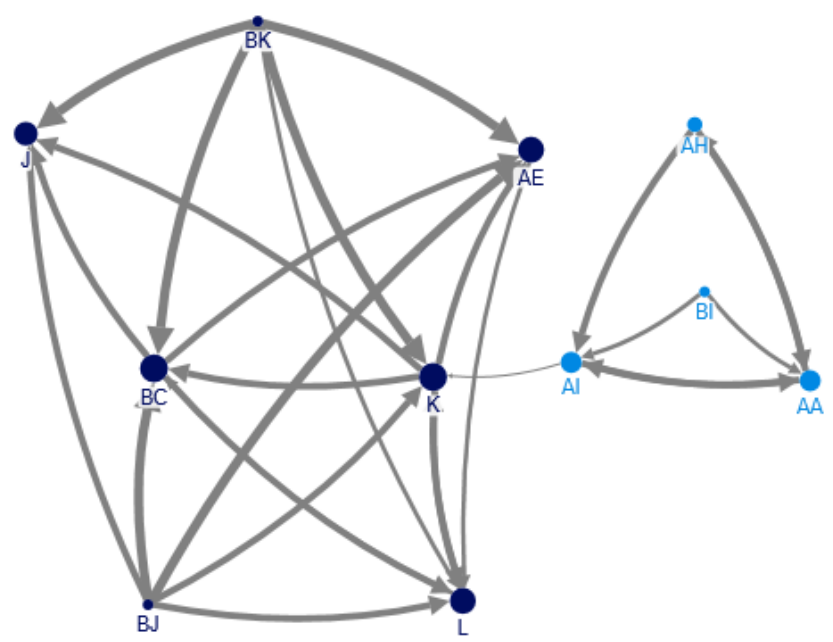

Figura 2. Sociograma de relaciones en "pedir consejo sobre cómo perfeccionar clases" para la Escuela Cielo

Fuente: Elaboración propia.

En cuanto la Escuela Tierra, el cuadro 4 revela un importante liderazgo de ambas jefaturas, ubicándose siempre entre los que reciben más nominaciones. Sin embargo, si bien otros docentes figuran de manera importante para el ámbito de "procesos de mejora en la disciplina", su participación va decreciendo prácticamente en la misma magnitud para las dimensiones "consejo" y "prácticas innovadoras". En esta última dimensión se encuentra el único caso de un docente que no realiza ninguna nominación y que a su vez solo recibe una (docente $\mathrm{A}$, de Matemáticas). Interesantemente, AX, jefe del Departamento de Matemáticas, va realizando cada vez menos nominaciones, en particular solo una en el ámbito de "obtención de prácticas innovadoras".

Cuadro 4. Indicadores de centralidad la Escuela Tierra

\begin{tabular}{llcccccc}
\hline \multirow{2}{*}{ NoDo } & \multirow{2}{*}{ CARGo } & \multicolumn{5}{c}{ DIMENSIONES DE COLABORACIÓN } \\
\cline { 3 - 7 } & & \multicolumn{2}{c}{ P. Mejora } & \multicolumn{2}{c}{ Consejo } & \multicolumn{2}{c}{ P. Innovadoras } \\
\cline { 3 - 7 } & & $\begin{array}{c}\text { In- } \\
\text { Degree }\end{array}$ & $\begin{array}{c}\text { Out- } \\
\text { Degree }\end{array}$ & $\begin{array}{c}\text { In- } \\
\text { Degree }\end{array}$ & $\begin{array}{c}\text { Out- } \\
\text { Degree }\end{array}$ & $\begin{array}{c}\text { In- } \\
\text { Degree }\end{array}$ & Out- \\
\hline AX & Jefatura Matemáticas & 9 & 7 & 6 & 1 & 6 & 4 \\
AA & Jefatura Lenguaje & 8 & 9 & 5 & 4 & 5 & 1 \\
D & Docente Lenguaje & 8 & 5 & 4 & 2 & 4 & 3 \\
V & Docente Matemáticas & 8 & 11 & 4 & 5 & 5 & 5 \\
A & Docente Matemáticas & 7 & 4 & 2 & 1 & 1 & 0 \\
AW & Docente Matemáticas & 7 & 3 & 4 & 3 & 3 & 4 \\
AK & Docente Lenguaje & 6 & 5 & 2 & 5 & 3 & 4 \\
L & Docente Lenguaje & 6 & 2 & 5 & 1 & 4 & 2 \\
G & Docente Matemáticas & 6 & 7 & 3 & 4 & 3 & 4 \\
AI & Docente Matemáticas & 6 & 5 & 4 & 4 & 4 & 3 \\
K & Docente Lenguaje & 3 & 9 & 1 & 5 & 3 & 5 \\
Z & Docente Lenguaje & 3 & 10 & 2 & 7 & 2 & 8 \\
\hline Funyyyyyy
\end{tabular}

Fuente: Elaboración propia.

De la figura 3, aparte de lo ya destacado para el docente A y la jefatura AX, cabe mencionar que el análisis nuevamente detecta dos clústeres claramente definidos, representando en 
azul oscuro a Lenguaje y azul claro a Matemáticas, este último presentando mayor frecuencia en sus interacciones. Se observa que los departamentos interactúan entre sí, pero en menor medida que en las otras dimensiones e incluso que en las otras escuelas analizadas. Sin embargo, es el caso del docente A el que llama la atención, pues como se ha mencionado, es un docente de Matemáticas, pero que interactúa con un colega de Lenguaje (Z), siendo su única conexión en la red referida a la "obtención de prácticas innovadoras".

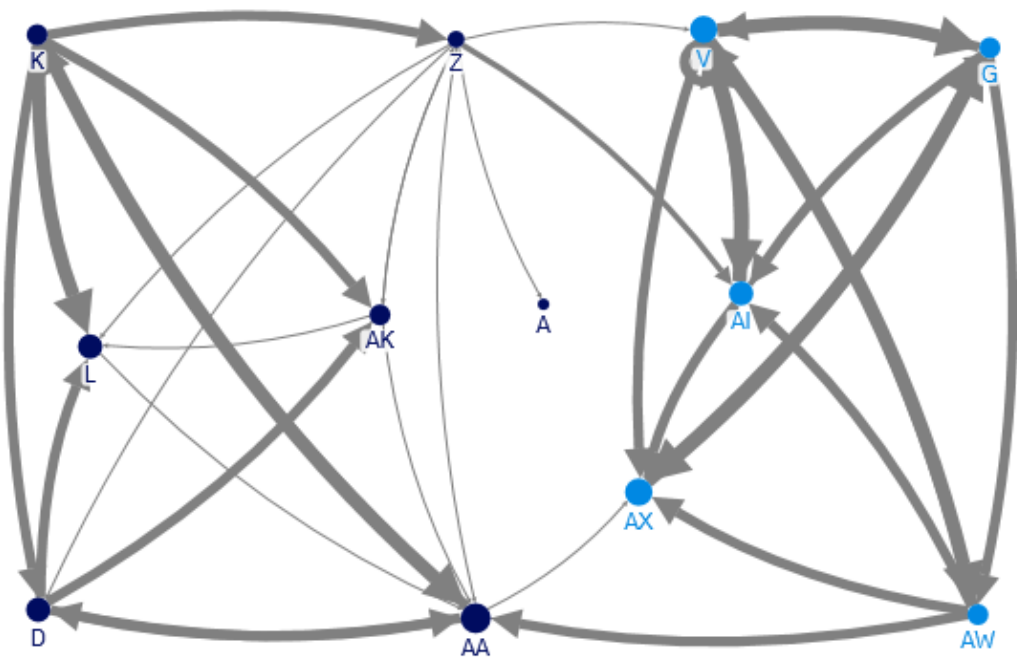

Figura 3. Sociograma de relaciones en "obtención de prácticas innovadoras" para la Escuela Tierra

Fuente: Elaboración propia.

\section{Discusión}

El presente estudio examinó el liderazgo docente en departamentos de Lenguaje y Matemáticas en centros escolares secundarios chilenos a través de un análisis de las interacciones entre las jefaturas de departamentos -vistos como líderes formales- y sus colegas. Utilizando la técnica de análisis de redes sociales se establecieron patrones de interacciones intra-departamentos y entre departamentos en función a tres dimensiones de colaboración entre docentes: a) a quién recurre, y con cuánta frecuencia, para trabajar en procesos de mejora escolar en su disciplina; b) a quién recurre, y con cuánta frecuencia, para pedir consejo sobre cómo mejorar su enseñanza; y c) a quién recurre, y con cuánta frecuencia, para obtener prácticas innovadoras.

Los resultados revelan tres hallazgos principales: a) se detectaron liderazgos múltiples en los departamentos; b) el liderazgo docente en las escuelas y departamentos analizados asume distintas configuraciones, según la dimensión de colaboración analizada; y c) la interacción entre docentes de distintos departamentos varía entre escuelas y se relaciona con la dimensión de colaboración analizada.

Un primer resultado refiere a que, en mayor o menor medida, en los seis departamentos estudiados se observó la co-existencia del liderazgo asociado al cargo formal de jefe de departamento y la distribución del liderazgo entre docentes. Bush, Yoon y Ng (2019) resumen varias investigaciones que concurren en señalar que la distribución del liderazgo 
no es incompatible con formas más jerárquicas de organización, siempre y cuando los líderes en cargos formales posibiliten esta distribución. Investigaciones previas sugieren que algunos docentes son reacios a asumir completamente la responsabilidad de tomar decisiones que son consideradas negativas por quienes se ven afectados por ellas (Little, 1995). Los docentes son más proclives a recurrir a aquellos que les expresen solo lo positivo o no cuestionen el status quo del departamento, en lugar de colegas que los desafían y presionen para que se transformen o mejoren. Por lo tanto, las normas implícitas o explícitas de igualitarismo, junto con las tensiones entre la autonomía docente y la no intervención que prevalecen en algunos departamentos, pueden ser obstáculos importantes para el ejercicio del liderazgo entre colegas para la mejora educativa y el cambio (Aubrey-Hopkins y James, 2002).

Las diferencias observadas no se pueden explicar a partir de los datos reportados en este estudio. No obstante, es importante reconocer que, como líderes medios, los jefes de departamento requieren involucrarse en procesos micropolíticos, al negociar la conciliación de intereses de la dirección escolar y de sus pares docentes. Para estos efectos, buscan generar consensos entre sus pares, sin imponer una visión (Cortez y Zoro, 2018). Las distribuciones de los procesos de influencia que se observaron en estas tres escuelas pueden estar dando cuenta de una estrategia micropolítica de los jefes de departamento (Flessa, 2009). Esta posible explicación amerita investigaciones respecto a factores que den cuenta de los patrones de vínculos profesionales detectados a través de la descripción que ofrece el ARS.

Un segundo resultado refiere a diferencias intra-escuela respecto de la densidad de las redes en función a la dimensión de colaboración sobre la cual se consulta a los docentes. Leithwood y Louis (1998) han postulado que las unidades departamentales pueden contribuir al aprendizaje a nivel individual, colectivo y organizacional. Un rol clave del jefe de departamento es articular estos tres niveles de aprendizaje. La evidencia recopilada en el presente estudio sugiere la presencia de ciertas regularidades respecto a cómo se abordan estos tres niveles en las dimensiones de colaboración e influencia mutua sobre las cuales los docentes fueron consultados. Los docentes reportan más interacciones respecto a procesos de mejora escolar, en comparación a interacciones para mejorar su enseñanza o innovar en sus prácticas pedagógicas. Considerando que las escuelas secundarias seleccionadas han presentado procesos de mejora sostenida en los últimos años, estos resultados corroboran la prioridad de este tipo de acciones colectivas. Sin embargo, aquellas interacciones que involucran algún tipo de transformación educativa, tales como perfeccionar clases u obtener prácticas innovadoras podrían ser menos frecuentes por tener un foco más individual. De ser así, potencialmente, la contribución de los departamentos al aprendizaje docente estaría limitada. Por otra parte, la escasez de interacciones en torno a la generación de prácticas innovadora puede presentar una limitante para el aprendizaje organizacional.

Stoll y Fink (1998) han descrito los desafíos al mejoramiento continuo en escuelas que logran ser efectivas y que, al no potenciar la innovación frente a cambios en las demandas sociales, no siempre logran adaptarse. Por otra parte, un estudio realizado en Canadá en nueve escuelas secundarias mostró que la estructura departamental era un factor que inhibía la capacidad de cambio (Ross y Hannay, 2001). Esos autores plantean que los departamentos tienden a representar culturas homogéneas, con pocos espacios para el cuestionamiento de lo que se ha construido como sentido común respecto del funcionamiento de sus escuelas. Más aun, la estructura departamental puede limitar el 
intercambio de ideas entre las distintas disciplinas del currículo escolar, impactando negativamente en el cambio organizacional.

Lo ya señalado refiere también al tercer resultado de este estudio, respecto de la densidad en las interacciones entre los profesores de Lenguaje y Matemáticas. Este resultado muestra la utilidad del ARS para estudiar la cultura escolar en la cual se moviliza el trabajo colectivo para la mejora. En la Escuela Agua, y en relación con el tema de procesos de mejora escolar, es donde observamos más interacciones entre departamentos y esto puede ser un factor que propicie la mejora escolar. Esta red muestra que, a pesar de sus diferencias curriculares, es relevante tener en cuenta a otros colegas más allá del propio departamento para trabajar y lograr la mejora educativa.

En la Escuela Cielo, y con relación a buscar consejos sobre cómo mejorar la enseñanza y obtener prácticas innovadoras, es donde se observan menos interacciones. En la Escuela Tierra, las interacciones se dan más entre docentes de la misma disciplina que entre los departamentos, ya que se observan dos clústeres distintivos, al igual que para la Escuela Cielo. Las redes de estas dos escuelas representan una limitación identificada por varios autores respecto de la estructura departamental. Siguiendo la tipología de culturas escolares propuesta por Hargreaves (2005), estas redes mostrarían una cultura escolar balcanizada, donde los departamentos disciplinarios parecieran haberse constituidos en subgrupos al interior de la organización escolar, con escasas oportunidades de interacción con otros departamentos.

La existencia de estas sub-redes representa un desafío para la mejora escolar cuando hay aislamiento y los subgrupos están separados por fronteras poco permeables, generando comunidades de práctica que difieren en valores, creencias y prácticas (Hargreaves, 2005). Las culturas balcanizadas tienen importantes consecuencias para la posibilidad de compartir una visión del proyecto educativo de la escuela, cuya materialización requiere del esfuerzo colectivo de la comunidad escolar. Los datos de este estudio no permiten comprender características de liderazgo o características organizacionales que puedan explicar los tipos de redes descritos, pero por los efectos negativos de una cultura escolar balcanizada estos resultados ameritan nuevos estudios.

\section{Conclusiones}

Una de las principales conclusiones de este estudio es la utilidad de la técnica de ARS para describir el capital social de una escuela y cómo fluyen ideas, información, recursos e influencia en los departamentos y entre departamentos (Daly, 2010). Una segunda conclusión concurre con lo señalado por otros autores respecto que el liderazgo, como una cualidad organizacional, no se limita a asignarlo formalmente (Ogawa y Bossert, 1995), ya que este necesita integrarse en las prácticas e interacciones de los docentes. Además, dada la evidencia empírica de los patrones de interacciones, la determinación del grado de distribución de una red de liderazgo no puede considerarse solamente como una cuestión de estructura o diseño organizacional. Una forma de abordar esta problemática es a través del análisis de las redes de docentes y determinar si sus patrones de centralidad y densidad, junto con los sociogramas y sus clústeres, adoptan o no configuraciones de liderazgo distribuido, así como el tipo de cultura organizacional en el cual funcionan los departamentos. 
En este sentido, este estudio hipotetizó que aquellas redes departamentales caracterizadas por una alta densidad de red y una centralidad moderada, es decir donde el jefe del departamento es tan relevante como otros colegas, serían ejemplos de departamentos cohesionados y con patrones de liderazgo distribuido. Sin embargo, los resultados muestran que las densidades de red y los indicadores de centralidad de las jefaturas de departamento están asociadas a la dimensión de colaboración que se puede generar entre docentes. Los patrones detectados en la distribución del liderazgo, especialmente en interacciones de búsqueda de consejos y prácticas innovadoras para la mejora educativa, muestran menor densidad en comparación a interacciones para abordar la mejora escolar. Esto puede tener implicaciones importantes para los tres niveles de aprendizaje que se requieren para la mejora escolar: individual, colectivo y organizacional (Leithwood y Louis, 1998).

El estudio, por su carácter descriptivo, no permite identificar factores que pueden moldear los patrones de red que se identificaron, como por ejemplo la carga de trabajo de los jefes de departamento, la antigüedad tanto como docentes como en el cargo, la historia de innovación de los departamentos, la perspectiva de género del cargo, entre otros factores. A su vez, estas conforman algunas de las limitaciones del estudio. Respecto a esto último, es posible ampliar la mirada a otras dimensiones de la mejora y cambio educativo, junto con tener en consideración mayor diversidad de tamaños tanto de los establecimientos educativos como de sus departamentos.

Wenner y Campbell (2017) sintetizan tres condiciones que inciden en el liderazgo de los jefes de departamentos y sus efectos en la mejora escolar: (a) las competencias para ejercer este tipo de liderazgo, (b) las prácticas de directivos escolares que generan condiciones para este liderazgo, y (c) la cultura profesional orientada a la colaboración y el mejoramiento. Las diferencias observadas entre escuelas y entre departamentos de una misma escuela sugiere la necesidad de investigar estas condiciones para comprender su incidencia en las redes detectadas. La hipótesis es que el tipo de redes que se establecen podrían estar ligadas a condiciones institucionales como las ya señaladas. Por último, la acotada muestra utilizada no permite generalizaciones, pero sí entrega importante evidencia para un tema poco estudiado tanto en Chile como en otras latitudes hispanoparlantes.

El liderazgo más o menos preponderante de un jefe de departamento se asocia a la definición clara, desde la dirección escolar, de las responsabilidades y tareas del cargo, junto al reconocimiento por su realización (Salleh, 2017). Esto, a su vez, implica que los directivos escolares comprenden a cabalidad cómo los líderes medios contribuyen a la mejora escolar y cómo ellos los pueden apoyar para ejercer procesos de influencia con sus pares. Las condiciones que genera el director o directora de una escuela secundaria para propiciar el liderazgo docente es otro aspecto que emerge como estudio futuro para comprender las variaciones observadas en las redes sociales descritas en la presente investigación.

\section{Agradecimientos}

Se agradece el financiamiento aportado por FONDECYT (Proyecto $\mathrm{N}^{\circ} 1180687$ ) y de CONICYT FB 003. 


\section{Referencias}

Araya, F. (2015). Rediseño del rol y función del jefe de departamento de asignatura. Estudio de caso (Tesis de maestría). Santiago de Chile: Pontificia Universidad Católica de Chile.

Aubrey-Hopkins, J. y James, C. (2002). Improving practice in subject departments: The experience of secondary school subject leaders in Wales. School Leadership and Management, 22(3), 305320. https://doi.org/10.1080/1363243022000020426

Barry, D. (1991). Managing the bossless team: Lessons in distributed leadership. Organisational Dynamics, 20(1), 31-47. https://doi.org/10.1016/0090-2616(91)90081-J

Bellei, C., Valenzuela, J., Vanni, X. y Contreras, D. (2014). Lo aprendí en la escuela. ¿Cómo se logran procesos de mejoramiento escolar?. Santiago de Chile: LOM.

Bennett, N. (1995). Managing professional teachers: Middle management in primary and secondary schools. Londres: Paul Chapman.

Bolívar, A. (2014). Building school capacity: Shared leadership and professional learning communities. A research proposal. International Journal of Educational Leadership and Management, 2(2), 147-175. https://doi.org/10.4471/ijelm.2014.15

Borgatti, S. P. y Everett, M. G. (2006). A graph-theoretic perspective on centrality. Social Networks, 28, 466-484. https://doi.org/10.1016/j.socnet.2005.11.005

Bush, T. (2003). Theories of educational leadership and management. Londres: Sage. https://doi.org/10.1177/0263211X0303102100

Bush, T., Yoon, A. y Ng, M. (2019). Distributed leadership and the Malaysia education blueprint enactment in a highly centralised context. Journal of Educational Administration, 57(3), 279295. https://doi.org/10.1108/JEA-11-2018-0206

Busher, H., Harris, A. y Wise, C. (2001). Subject leadership and school improvement. Londres: Paul Chapman.

Camburn, E., Rowan, B. y Taylor, J. E. (2003). Distributed leadership in schools: The case of elementary schools adopting comprehensive school reform models. Educational Evaluation and Policy Analysis, 25(4), 347-373. https://doi.org/10.3102/01623737025004347

Cortez, M. y Zoro, B. (2016). El rol estratégico de los liderazgos medios al interior de las escuelas para la mejora escolar: una mirada desde la literatura internacional. Recuperado de https://www.lidereseducativos.cl/wp-content/uploads/2017/01/NT-8.pdf

Day, C. y Harris, A. (2002). Teacher leadership, reflective practice, and school improvement. En K. Leithwood (Coord.), Second international handbook of educational leadership and administration (pp. 957-955). Dordrecht: Springer. https://doi.org/10.1007/978-94-010-0375-9_32

Daly, A. J. (2010). Social network theory and educational change. Cambridge, MA: Harvard University Press.

Daly, A. J., Moolenaar, N. M., Bolivar, J. M. y Burke, P. (2010). Relationships in reform: The role of teachers' social networks. Journal of Educational Administration, 48(3), 359-391. https://doi.org/10.1108/09578231011041062

De Lima, J. A. (2008). Department networks and distributed leadership in schools. School Leadership and Management, 28(2), 159-187. https://doi.org/10.1080/13632430801969864

Downey, C. (2018). Utilizing social network analysis to identify the structural features of teachers' knowledge and resource sharing networks within schools. Profesorado, Revista de Currículum y Formación del Profesorado, 22(2), 133-159.

https://doi.org/10.30827/profesorado.v22i2.7718 
Flessa, J. (2009). Educational micropolitics and distributed leadership. Peabody Journal of Education, 84, 331-349. https://doi.org/10.1080/01619560902973522

Flückiger, B., Lovett, S., Dempster, N. y Brown, S. (2015). Middle leaders: Career pathways and professional learning needs. Leading and Managing, 21(2), 60-74.

Friedkin, N. E. y Slater, M. R. (1994). School leadership and performance: A social network approach. Sociology of Education, 14(2), 139-157. https://doi.org/10.2307/2112701

Frost, D. y Harris, A. (2003). Teacher leadership: Towards a research agenda. Cambridge Journal of Education, 33(3), 479-498. https://doi.org/10.1080/0305764032000122078

Garay, S., Queupil, J. P., Maureira, O., Guiñez, C. y Garay, C. (2019). El liderazgo desde la perspectiva del análisis de redes: Una experiencia en escuelas vulnerables y efectivas de Chile. Profesorado, Revista de Currículum y Formación del Profesorado, 23(2), 169-188. https://doi.org/10.30827/profesorado.v23i2.9269

Gronn, P. (2002). Distributed leadership. En K. Leithwood (Coord.), Second international handbook of educational leadership and administration (pp. 653-696). Dordrecht: Springer. https://doi.org/10.1007/978-94-010-0375-9_23

Hadfield, M. y Jopling, M. (2012). How might better network theories support school leadership research? School Leadership \& Management, 32(2), 109-121. https://doi.org/10.1080/13632434.2012.670115

Hallinger, P. y Heck R. H. (2014). Liderazgo colaborativo y mejora escolar: Comprendiendo el impacto sobre la capacidad de la escuela y el aprendizaje de los estudiantes. REICE. Revista Iberoamericana sobre Calidad, Eficacia y Cambio en Educación, 12(4e), 71-88.

Hargreaves, A. (2005). Colaboración y colegialidad artificial (copa reconfortante o cáliz envenenado). En A. Hargreaves (Ed.), Profesorado, cultura y postmodernidad (pp. 210-234). Londres: Ediciones Morata.

Harris, A. (2000). Effective leadership and departmental improvement. Westminster Studies in Education, 23, 81-90. https://doi.org/10.1080/0140672000230108

Harris, A. (2001). Department improvement and school improvement: A missing link? British Educational Research Journal, 27(4), 477-486. https://doi.org/10.1080/01411920120071470

Harris, A. (2003). Teacher leadership as distributed leadership: Heresy, fantasy or possibility? School Leadership and Management, 23(3), 313-324. https://doi.org/10.1080/1363243032000112801

Harris, A. (2004). Distributed leadership and school improvement: Leading or misleading? Educational Management Administration $\Xi^{2}$ Leadership, 32(1), 11-24. https://doi.org/10.1177/1741143204039297

Harris, A. (2013). Distributed school leadership: developing tomorrow's leaders. Londres: Routledge. https://doi.org/10.4324/9780203607909

Hopkins, D., Ainscow, M. y West, M. (1994). School improvement in an era of change. Londres: Cassell.

Hoppe, B. y Reinelt, C. (2010). Social network analysis and the evaluation of leadership networks. The Leadership Quarterly, 21(4), 600-619. https://doi.org/10.1016/j.leaqua.2010.06.004

Leithwood, K. y Louis, K. S. (1998). Organizational learning in schools: An introduction. En K. Leithwood y K. S. Louis (Eds.), Organizational learning in schools (pp. 1-14). Lisse: Swets \& Zeitlinger. 
Leithwood, K., Day, C., Sammons, P., Harris, A. y Hopkins, D. (2006). Seven strong claims about successful school leadership. Londres: NCSL.

Little, J. W. (1995). Contested ground: The basis of teacher leadership in two restructuring high schools. The Elementary School Journal, 96(1), 47-62. https://doi.org/10.1086/461814

Martínez-Valdivia, E., García-Martínez, I. e Higueras-Rodríguez, M. L. (2018). El liderazgo para la mejora escolar y la justicia social. Un estudio de caso sobre un centro de educación secundaria obligatoria. REICE. Revista Iberoamericana sobre Calidad, Eficacia y Cambio en Educación, 16(1), 35-51. https://doi.org/10.15366/reice2018.16.1.003

Merriam, S. B. (1988). Case study research in education: A qualitative approach. San Francisco, CA: Jossey-Bass.

Moolenaar, N. M. (2012). A social network perspective on teacher collaboration in schools: Theory, methodology, and applications. American Journal of Education, 119(1), 7-39. https://doi.org/10.1086/667715

Murillo, F. J. y Krichesky, G. J. (2015). Mejora de la escuela: Medio siglo de lecciones aprendidas. REICE. Revista Iberoamericana sobre Calidad, Eficacia y Cambio en Educación, 13(1), 69-102.

Ogawa, R. T. y Bossert, S. T. (1995). Leadership as an organizational quality. Educational Administration Quarterly, 31(2), 224-243. https://doi.org/10.1177/0013161X95031002004

Robinson, V. M. (2007). School leadership and student outcomes: Identifying what works and why. Australian Council for Educational Leaders, 41, art 5.

Ross, J. y Hannay, L. (2001). Internalizing change capacity in secondary schools through organizational change. Alberta Journal of Educational Research, 47(4), 325-340.

Salleh, H. (2017). The attractions and challenges of building teacher leaders. Comunicación presentanda en la Conferencia BELMAS 2017. Stratford-Upon-Avon, Reino Unido.

Scott, J. (2000). Social network analysis: A handbook. Thousands Oaks, CA: Sage.

Siskin, L. S. y Little, J. W. (1995). The subjects in question: Departmental organization and the high school. Nueva York, NY: Teachers College Press.

Smith, M., Ceni, A., Milic-Frayling, N., Shneiderman, B., Mendes Rodrigues, E., Leskovec, J. y Dunne, C. (2010). NodeXL: A free and open network overview, discovery and exploration add-in for Excel 2007/2010. Recuperado de http://nodexl.codeplex.com/

Smith, P. S., Trygstad, P. J. y Hayes, M. L. (2018). Social network analysis: A simple but powerful tool for identifying teacher leaders. International Journal of Leadership in Education, 21(1), 95-103. https://doi.org/10.1080/13603124.2016.1195016

Spillane, J. P. (2006). Distributed leadership. San Francisco, CA: Jossey-Bass.

Stoll, L. y Fink, D. (1998). The cruising school: The unidentified ineffective school. En L. Stoll y K. Myers (Eds.), No quick fixes (pp. 189-206). Londres: Falmer Press.

Valenzuela, J. P., Bellei, C. y Allende, C. (2016). Measuring systematic long-term trajectories of school effectiveness improvement. School Effectiveness and School Improvement, 27(4), 473491. https://doi.org/10.1080/09243453.2016.1150861

Wakita, K. y Tsurumi, T. (2007). Finding community structure in mega-scale social networks. Recuperado de https://dl.acm.org/doi/10.1145/1242572.1242805

Wallace, M. (2002). Modelling distributed leadership and management effectiveness: Primary school senior management teams in England and Wales. School Effectiveness and School Improvement, 13(2), 163-186. https://doi.org/10.1076/sesi.13.2.163.3433 
Wang, A. H., Walters, A. M. y Thum, Y. M. (2013). Identifying highly effective urban schools: Comparing two measures of school success. International Journal of Educational Management, 27(5), 517-540. https://doi.org/10.1108/09513541311329878

Wasserman, S. y Faust, K. (1994). Social network analysis: Methods and applications. Cambridge: Cambridge University Press. https://doi.org/10.1017/CBO9780511815478

Wenner, J. A. y Campbell, T. (2017). The theoretical and empirical basis of teacher leadership: A review of the literature. Review of Educational Research, 87(1), 134-171.

https://doi.org/10.3102/0034654316653478

\section{Breve CV de los autores}

\section{Juan Pablo Queupil}

Doctor en Liderazgo Educacional y Educación Internacional Comparada de The Pennsylvania State University, Estados Unidos. Actualmente es académico e investigador del "Centro de Investigación en Educación y Aprendizaje Basado en la Comunidad" de la Universidad Católica Silva Henríquez. Sus intereses de investigación incluyen: gestión y liderazgo educacional; colaboración e internacionalización de la investigación en educación; perspectiva de género; inclusión y justicia educacional; trayectorias y transiciones estudiantiles; educación superior; y política educacional. A su vez, es parte de la actual directiva de la "Red de Investigadores en Educación Chilena" (RIECH). ORCID ID: https://orcid.org/o000-0002-7324-9275. Email: jqueupil@ucsh.cl

\section{Carmen Montecinos}

Doctora en Psicología Educacional de la Southern Illinois University-Carbondale. Profesora Titular de la Escuela de Psicología de Valparaíso y Directora Ejecutiva del Centro de Liderazgo para la Mejora Escolar de la Pontificia Universidad Católica de Valparaíso e Investigadora Principal del CIAE, Universidad de Chile. Su investigación y publicaciones abordan temáticas de desarrollo y aprendizaje en los profesionales de la educación y mejora escolar. ORCID ID: https://orcid.org/0000-0001-8382-1848. Email: carmen.montecinos@pucv.cl 